\title{
Cefotiam Hydrochloride
}

National Cancer Institute

\section{Source}

National Cancer Institute. Cefotiam Hydrochloride. NCI Thesaurus. Code C65302.

The hydrochloride salt form of cefotiam, a third-generation, semi-synthetic, beta-lactam cephalosporin antibiotic with antibacterial activity. Cefotiam binds to penicillin-binding proteins (PBPs), transpeptidases that are responsible for crosslinking of peptidoglycan. By preventing crosslinking of peptidoglycan, cell wall integ rity is lost and cell wall synthesis is halted. 